\title{
A Model Study on Classified Compensation for Abnormal Response Following Immunization
}

\author{
Shanshan $\mathrm{Mei}^{1,}$ a \\ ${ }^{1}$ Xi'an Medical University, Xi'an, China \\ a105263231@qq.com
}

Keywords: Abnormal response; Category 1 vaccine; Category 2 vaccine; Compensation

\begin{abstract}
China has a classified compensation mechanism for Category 1 and Category 2 vaccines when abnormal response occurs. This mechanism gives rise to fairness problems because there should be no compensational difference between Category 1 and Category 2 vaccines, thus calling for a new compensational standard. This paper makes a discussion on the principle and standard for reclassifying vaccines and gives suggestions on the compensational model for abnormal responses.

2005 witnessed the enactment of Regulations on the Management of Vaccine Circulation and Vaccination by the Chinese government. It classified all vaccines into two categories: Category 1, free of charge, inoculated by citizens according to the requirements of the governments, includes vaccines on the State Immunization Programs, the added vaccines by provinces, autonomous regions and municipalities when implementing the National Immunization Programs, as well as vaccines for contingency vaccination by People's government above the county level or health administrative sectors, and vaccines for Mass Vaccination. Category 2 refers to vaccines that are not free, and residents can choose whether they want to be inoculated.

Once abnormal response occurs following vaccination, compensations are different on the basis of the categories. As for abnormal response caused by Category 1, its compensation, a one-time compensation, comes from Financial Departments under the People's Governments of Provinces, Autonomous Regions and Municipalities' vaccination expenses. Abnormal response caused by Category 2, its compensation is covered by vaccine companies.
\end{abstract}

\section{Questions}

The State Council has been adjusting policies and schemes on vaccines circulation and vaccination. In terms of vaccination program, the National Health and Family Planning Commission ruled that from May. $1^{\text {st }}, 2016$, a new poliomyelitis vaccine has been adopted, putting an end to trivalent oral polio vaccine (tOPV). tOPV had been replaced by bOPV, which is included on the National Immunization Programs with the first dose changed to injection from oral administration. The procedure for poliomyelitis vaccine immunization is adjusted to: a dose of inactivated vaccine to be inoculated by the age of two months; a dose inoculated by the three months, four months and four years old respectively.

The implementing of the scheme caused people's questioning over vaccine classification. Take poliomyelitis vaccine for example, the first dose is inactivated vaccine (RMB 198), and it has been included on the National Immunization Programs with the government paying for the expense. The second, third and fourth dose are still OPV, if refused by inoculators or their guardians, can be changed to inactivated vaccine with citizens covering the expense. When there is an abnormal response following vaccination, the first dose, the vaccine on the National Immunization Programs, is compensated by the state. The other three, inactivated vaccines at citizens' own expense, are compensated by vaccine companies. The same vaccine at different vaccination time could have different compensation procedure and compensators following abnormal response, is it fair to inoculators? 


\section{Why should Category 1 and Category 2 Vaccines be Treated Equally Following Abnormal Response?}

From the Perspective of Human Rights. Inoculators are endowed with personal rights, including the right to health and life. When Category 2 vaccine has an abnormal response, a probable damage to the organs or executive functions, citizens would claim a compensation from vaccine companies through tortuous legal procedures while suffer from both physical pains and mental distress, but their efforts may possibly be echoed with a little or even little compensation. Category 1 vaccine, however tortuous in claming compensation, is at least guaranteed by the national finance.

Category 1 and Category 2 vaccines, under some circumstances, are targeted at the same disease, or even they are the same vaccines like the first dose of poliomyelitis vaccine(free of charge) and the second, third and fourth dose of poliomyelitis vaccine (at citizens' own expense). The only difference between the above two lies in its vaccination time, leading to different compensation mechanisms and results following abnormal response, which violates the principle concerning human rights protection and relief.

With China respecting and protecting human rights, all Chinese citizens enjoy the rights to material assistance from the state and society when they are getting old, sick and unable to work. In a democratic society under the rule of law, everyone enjoys the right to equal protection under the law, and shares the social responsibility equally. Vaccination itself is public health services serving for public interests. If an individual or some citizens undertake some special obligation or suffer from severe harm, his/their loss should be shared by all the citizens through special relief measures, letting the public share the burden equally. From the perspective of human rights protection, when people's rights are abused, it is how to reinstate their rights though relief rather than treating them differently that should be considered.

Theory of Recompense. The Theory of Recompense writes that innocent harm and pains should be paid with sympathy and compensation. Based on this theory, the one who enjoys interests bears risks. Vaccination has risks, with the rate of abnormal response ranging from 1/10000000 to 1/100000. This risk should be shared by the public, the beneficiaries. The state stands for the national and public interests, therefore, it should compensate those who suffer from impaired tissues and death out of abnormal response following vaccination.

Though people inoculating Category 2 vaccine aims at preventing diseases, their vaccination serves the benefits of the majority of the community as a whole. Take hepatitis B vaccine(belongs to Category 2) as an example, antibodies are produced after vaccination, preventing the spread of HBV in the body, narrowing its range, thus contributing to the public. To conclude, Category 2 vaccines are as important to people's health as Category 1 vaccines as far as the national interests concerned. Therefore, citizens with abnormal response following the vaccination of Category 2 vaccines should be compensated by the national finance as well.

\section{Suggestions for Vaccine Classification}

In view of the above analysis, abnormal response following Category 1 and Category 2 vaccines should be treated equally but it is with difficulty in real life. First, how much will the state compensate is closely linked to the affluence of the country. With the country being wealthy, people with all abnormal responses after vaccination can get compensation, otherwise, only some abnormal responses can be compensated. Second, Category 2 vaccines vary from one another, for example, Influenza Vaccine inoculation is far less necessary than HBV inoculation. Therefore, for some diseases with less harm, it is unnecessary for the state to treat their vaccines with vaccines preventing epidemic, detrimental diseases in the same way.

How should we ensure compensational fairness while avoid problems caused by the lack of differentiation between Category 1 and 2 vaccines? This paper suggests that all the problems could be solved when we properly the vaccine classification. 
The Principle for Vaccine Classification: Whether to be Inoculated with Enforcement. Charge-free and inoculation with enforcement decide vaccine classification. As charge-free serves as a means to enforce inoculation, the author holds that the necessity of enforcement should be the first factor in deciding classification. Vaccination is for the need to prevent and control infectious diseases. The present Category 1 vaccines are for epidemic, detrimental diseases, so they have to be inoculated with priority and enforcement. To safeguard public health, the number of people being inoculated should account for $85 \%$ of the total population. The state requires all Children in kindergarten and school to get vaccination (free of charge) according to the National Immunization Program. Therefore, the necessity of enforcement should be the first factor in deciding classification.

\section{Suggestions for Vaccine Classification}

Theoretical Basis for Classification. Vaccination proves to be an effective way to prevent infectious diseases. China's Law on the Prevention and Control of Infectious Diseases, after referring to the international standards on vaccine classification, categorizes 37 kinds of legally-managed contagious diseases into Class A, B and C, according to their ways, speed of transmission, intensity of epidemic process and harmfulness to the society. Vaccination should be conducted in accordance with the vaccination scheme and the necessity to prevent and control contagious diseases.

Vaccine classification should be in line with the necessity to prevent and control contagious diseases. Epidemics with great hazardousness should be on the list of forcible immunization.

New Standards for Classification. In ensuring epidemics with great hazardousness being prevented and controlled on a timely basis, vaccine classification must be in correspondence with the class of diseases. That is: Category 1 vaccines are for Class $\mathrm{A}$ and $\mathrm{B}$ diseases that are immunized forcibly by the state. Category 2 vaccines are for Class $\mathrm{C}$ diseases with citizens getting vaccination on their free will.

For Category 1 vaccines, the state decides the range of free-charge vaccines on the basis of regional economic development, meanwhile, respects citizens' free will to choose self-paying vaccines or free-charge vaccines.

\section{Recommendations for the Compensation Model on Abnormal Response Following Vaccination}

Different Methods Adopted Upon Different Types of Vaccines.The first type vaccines refer to those who can prevent the category B and category A infectious diseases. This kind of vaccine belongs to the compulsory one without taking its charges into account. The range for free depends on a country's economic development and on this basis, a country needs to respect people's independent choices. People can choose either the free-vaccine or the self-financed vaccine to finish the inoculation. If an abnormal effect happens to this kind of vaccine, the country will compensate for people's loss.

The second type vaccines refer to those who can prevent the category $\mathrm{C}$ infectious disease. People can inoculate this kind of vaccine on their own willing. Provided that this kind of vaccine reacts abnormally, the producer will bear the loss.

B Easing a country's burden for compensation through various channels

Firstly, setting up a compensation fund for abnormal vaccine reactions is recommended. The country is suggested to charge a certain fee from each vaccine selling to people and gathers them up to be the compensation funds. As there is any abnormal reaction of a vaccine, a part of the costs can be compensated by the fund and its interests.

Secondly, purchasing insurance makes sure the social security institutes undertake the loss produced by the vaccine's abnormal reaction. For instance, according to the "Tort Liability Law", the risk of the liability subject in the motor vehicle damages will be lowered by the social insurance institution to give the victims more compensation. In terms of the vaccine's abnormal reaction, the 
insurance company can undertake the compensation through purchasing insurance by the government.

Beijing has conducted a trial on this method. According to the principal of Beijing PQCC, "at present, the first type vaccine is compensated by the provincial finance. After a year's fully demonstration and research, the government brings into the insurance company by procurement. Then, the abnormal reaction, the solving measures and the compensation will be undertaken by the insurance company".

\section{Conclusion}

Vaccination is implemented for unspecific social public interests. On one hand, this measure benefits most of people objectively, on the other hand, it brings damages to a very few people caused by abnormal reactions. To be specific, a few people sacrifice their interests for the most. That's to say, everyone is a potential beneficiary, also a potential victim before the arrival of the damage caused by vaccine's abnormal reaction.

A well-working compensation system for vaccination can not only make up for a loss of the victim, but boost the public's confidence to the vaccination thus encouraging them to support this work. In order to establish a new standard characterized by entity-based classification and coerciveness without minding its free of charge or self-finance, "The Ordinance on the Management of Vaccine and Immunization" should accord with the social host law. Based on the newly regulated class, the country should construct the compensating mechanism through various channels in order to protect the victims' rights and interests suffered from abnormal reactions.

Certainly, there are many problems to be solved regarding the compensation of vaccines' abnormal reactions. First, our country is lack of a unified and specific procedure in the compensation of the vaccination's damage. Second, there is not a unified compensation range and standard. In addition, problems caused by the ordinances appear including a lack of a specific compensation standard, a great varying of the compensation standard and the misjudgments of the vaccine damage being seen everywhere. Moreover, much strictness is put on the reason of vaccines' abnormal reactions. Some vaccines' abnormal reactions and their mechanisms can't be explained by the present scientific knowledge. Judging the vaccine's damage through a medical-scientific causal relationship is quite strict to the victims for they will bear the risk of the unknown vaccine's abnormal reaction. Of course, these problems mentioned above need a lot of investigations and demonstrations, which is also a guiding direction for my later research.

\section{References}

[1] Shang Peng -hui, Liang Xiao-feng. Compensation of Adverse Events Following Immunization in China and Abroad, Chinese Journal Of Vaccines And Immunization[J] Vol.13 No.5 ,2007:487-490

[2] Yue Da-hai,Chang Jie,Hou Zhi-yuan. References on International Vaccination Injury Compensation Programs, Chinese Health Economics[J], 2014,33(1):93-96

[3] Chang Jie ,Hou Zhi-yuan,Yue Da-hai. Factors related to self-paid vaccination and its related factors among children aged 0-3 years in China ,Chin J Public Health[J], May 2014 Vol.30 No.5:579-582

[4] FENG Guozhong, MA Libin, MA Aixia. Enlightenment of Vaccine Injury Compensation System in the USA, China Pharmacy[J],2007,Vol.18No.1:15-16

[5] YUAN Ping, ZHENG Jing-shan, CAO Lei, Analysis of Category II Vaccine Utilization using Data from the Immunization Monitoring System in China2013, Chinese Journal Of Vaccines And Immunization[J] Vol.20 No.6, 2014:499-504 